\title{
Análisis territorial de los campamentos de México
}

\author{
Leonardo Nava Jiménez* \\ Oscar Frausto Martínez \\ Universidad de Quintana Roo
}

\section{Resumen}

Los campamentos son entes empresariales cuya peculiaridad sine qua non es su operación en un entorno natural, pero además conllevan diversas interacciones sociales y económicas relevantes en el paradigma de la sustentabilidad. En México son escasos los estudios enfocados a los campamentos y a las industrias que de ellos se derivan. Concibiendo el fenómeno turístico como un sistema de actores interrelacionados. El objetivo de esta investigación es identificar y caracterizar a los actores del sistema de campamentos en México por medio de sus atributos espaciales. La identificación se realizó con base en algunos directorios del sector (el de la Secretaría de Turismo, el de la Asociación Mexicana de Campamentos y el de la International Camping Fellowship) y en otros promocionales elaborados por México Desconocido y yumping.com.mx; la caracterización se llevó a cabo con los datos arrojados por la encuesta dirigida a los directivos de las empresas identificadas previamente, y mediante el análisis espacial de sus relaciones con elementos naturales y antropogénicos. Los resultados se discutieron y corroboraron con visitas a los sitios y con entrevistas a individuos clave en el sector. Se identificaron 519 actores, entre quienes predominan los discursos formativo y de desarrollo económico.

\section{Palabras clave}

Campamentos, México, estructura territorial, gestión del espacio, turismo sustentable.

*Correo electrónico: leonavaj@gmail.com, fraustomartinezoscar@gmail.com

Recibido: 30/03/2014 · Aceptado: 25/04/2014 


\title{
Analysis of the territorial structure of Camps in Mexico
}

\author{
Leonardo Nava Jiménez* \\ Oscar Frausto Martínez \\ Universidad de Quintana Roo
}

\begin{abstract}
Camps are businesses whose essential feature is their operation in a natural environment, while involving different social and economic interactions relevant in the sustainability paradigm. In México, the studies focused on camps and related industries are scarce. Conceiving the tourist phenomenon as a system of interrelated actors, the objective of this research is to identify and characterize the actors of the Mexican camping system by their spatial attributes. These actors were identified based on various industry directories: Sectur, Asociación Mexicana de Campamentos, International Camping Fellowship; and promotional directories such as México Desconocido and Yumping.com.mx. The characterization was developed from data obtained from the survey of camp managers, and based on the spatial analysis of their relations with natural and anthropogenic elements. The results were corroborated through in site visits and discussed in interviews with 519 identified key actors among whom the formative and the economic development discourse are predominant.
\end{abstract}

\section{KEY WORDS}

Camps, Mexico, territorial structure, space management, sustainable tourism.

*E-mail:leonavaj@gmail.com, fraustomartinezoscar@gmail.com 


\section{Introducción}

La noción inicial del campamento se ha transformado y diversificado, ha pasado de ser un concepto referente al espacio abierto ocupado de forma temporal y con fines principalmente militares (Varela Merino, 2009) a ser un término que, de acuerdo con el contexto o el adjetivo que lo acompañe, describe una amplia variedad de espacios: campamento prehistórico (Asouti, 2003), de turismo (Aparicio, 1999) o militar (Varela Merino, 2009); o de actividades: campamento de vacaciones (Lee Nolan y Broxon, 2000), organizado (Ramsing, 2007) o temático (Luján Ferrer y Rodríguez Sánchez, 2011), etcétera.

A partir de la Segunda Guerra Mundial, sobre todo en Estados Unidos, Canadá y Europa, los campamentos comenzaron a popularizarse como actividad recreativa (Lee Nolan y Broxon, 2000). Desde entonces, los campamentos con fines recreativos se han consolidado dentro de la actividad turística, al grado de ser estudiados, reconocidos y promovidos en la actualidad como un segmento importante del turismo en países y regiones como Estados Unidos, Canadá, Australia, Nueva Zelanda, Europa Occidental (Heritage Appreciation Unit, 2006; Janiskee, 1998; McEwen, 1986; Feo Parrondo, 2003) y, en años recientes, el sureste asiático (Brooker y Joppe, 2013).

En México, la aparición de los campamentos recreativos fue más tardía y se dio en menor escala. Apenas para 1990 se estimaban poco menos de 50 campamentos establecidos en todo el territorio nacional (Ortiz, 1994). Sin embargo, ya se planteaba la necesidad de desarrollar asociaciones, estudios y políticas que estimularan su desarrollo como estrategia para la recreación de la población y como atractivo turístico (Vergara, 1994; Ortiz, 1994).

Así, los campamentos en cuanto actividad recreativa se han integrado al sector turístico en México. Hoy en día existen:

- Directorios comerciales: Campamentosmexico.com (Mexplora, s.f.); México Desconocido (agosto 2005); yumping.com.mx (s.f.);

- asociaciones: Asociación Mexicana de Campamentos (Amc, 2013); International Camping Fellowship (ICF, 2013);

- regulaciones especializadas en este tipo de empresas: Reglamento de establecimientos de hospedaje, campamentos y paraderos de casas ro- 
dantes (rehcp) (Presidencia de la República, 1984); Norma Oficial Mexicana nom-06-tur-2009, Requisitos mínimos de información, higiene y seguridad que deben cumplir los prestadores de servicios turísticos de campamentos (Sectur, 2010);

- así como un registro de los establecimientos en la Secretaría de Turismo: Directorio de Prestadores de Servicios Turísticos de Naturaleza (dpstn) (Sectur, 2013).

No obstante, el modelo empresarial del campamento se concibe de formas diversas dependiendo del contexto.

Pese a su crecimiento y especialización, son escasos los estudios que abordan el tema en el país, y ninguno permite conocer la magnitud y las características de este tipo de empresas en el marco de la oferta turística nacional. Una estrategia empleada para bosquejar la situación de este segmento en diferentes países es el análisis territorial de sus actores, lo que facilita identificar sus relaciones espaciales con los recursos naturales, núcleos de población, organismos de gestión pública, vías de comunicación, entre muchos otros elementos del entorno (Feo Parrondo, 2003; Leefers y Vasievich, 1999; López Palomeque, 1988; Miranda Montero, 1985).

El sistema turístico se concibe como una red de actores interrelacionados que tienen un soporte físico en elementos infra- y supraestructurales (Hiernaux, 1989). Este trabajo se propone identificar a los actores que componen la oferta del sistema de campamentos en México, y caracterizarlos con base en el estudio de sus relaciones espaciales con los elementos del entorno.

\section{Metodología}

Para el logro del objetivo fue necesario:

a) Compilar información sobre la cantidad de actores involucrados en la industria de campamentos en México. Para ello se recurrió a cinco directorios especializados (AMC; yumping.com.mx; IcF; México Desconocido; Sectur), de los cuales se pudo generar una base de datos georreferenciada, cuyo nivel de resolución espacial es la localidad. 
b) Aplicar una encuesta electrónica a los representantes de los campamentos identificados. El instrumento se generó con el servicio de formularios de Google Drive y contiene los siguientes apartados: identificación del encuestado y de la organización que opera el campamento, actividades y servicios, valoración del mercado, funcionamiento interno y descripción de la sede. La encuesta se aplicó del 29 de octubre al 11 de noviembre de 2013.

c) Visitar siete campamentos y entrevistar a 13 actores clave, con el fin de complementar, corroborar y discutir los resultados obtenidos en los directorios y encuestas. Como evidencias se tienen las bitácoras de campo, el registro fotográfico y la revisión del discurso.

d) Realizar un análisis espacial con base en la distribución de los campamentos en México. Para ello se construyó un sistema de información geográfica utilizando el software ArcGIS 9.3, útil para establecer (a nivel estatal, municipal y local) las variables topográficas, climáticas, hidrológicas, áreas naturales protegidas (ANP) y paisajes; así como el contexto de las áreas urbanas y el sistema nacional de carreteras (a escala 1:250,000 de la base de datos vectoriales de la Comisión Nacional de Áreas Naturales Protegidas [Conanp, 2013]).

\section{Resultados}

Los resultados se han dividido en dos secciones: identificación y distribución territorial, y caracterización de los campamentos en México.

\section{Identificación y distribución}

Se identificaron 519 actores o campamentos distribuidos en 363 localidades, correspondientes a 262 municipios en 31 entidades federativas (gráfica 1). Los estados de Veracruz, México, Oaxaca y Puebla encabezan la actividad, en contraste con Sinaloa, Coahuila y Guerrero. En el nivel municipal, la actividad se concentra en Jalcomulco (Veracruz), Ensenada (Baja California), Santiago (Nuevo León), Puebla (Puebla), Ciudad Valles (San Luis Potosí) y Valle de Bravo (México). En la 
escala de localidad, Jalcomulco (14), Ciudad Valles (7) y Zapopan (5) presentan la mayor concentración de actores (figura 1). En el territorio nacional se puede reconocer un primer patrón básico de distribución en la zona centro y centro occidente de México, así como en el noroeste (Baja California y Sonora).
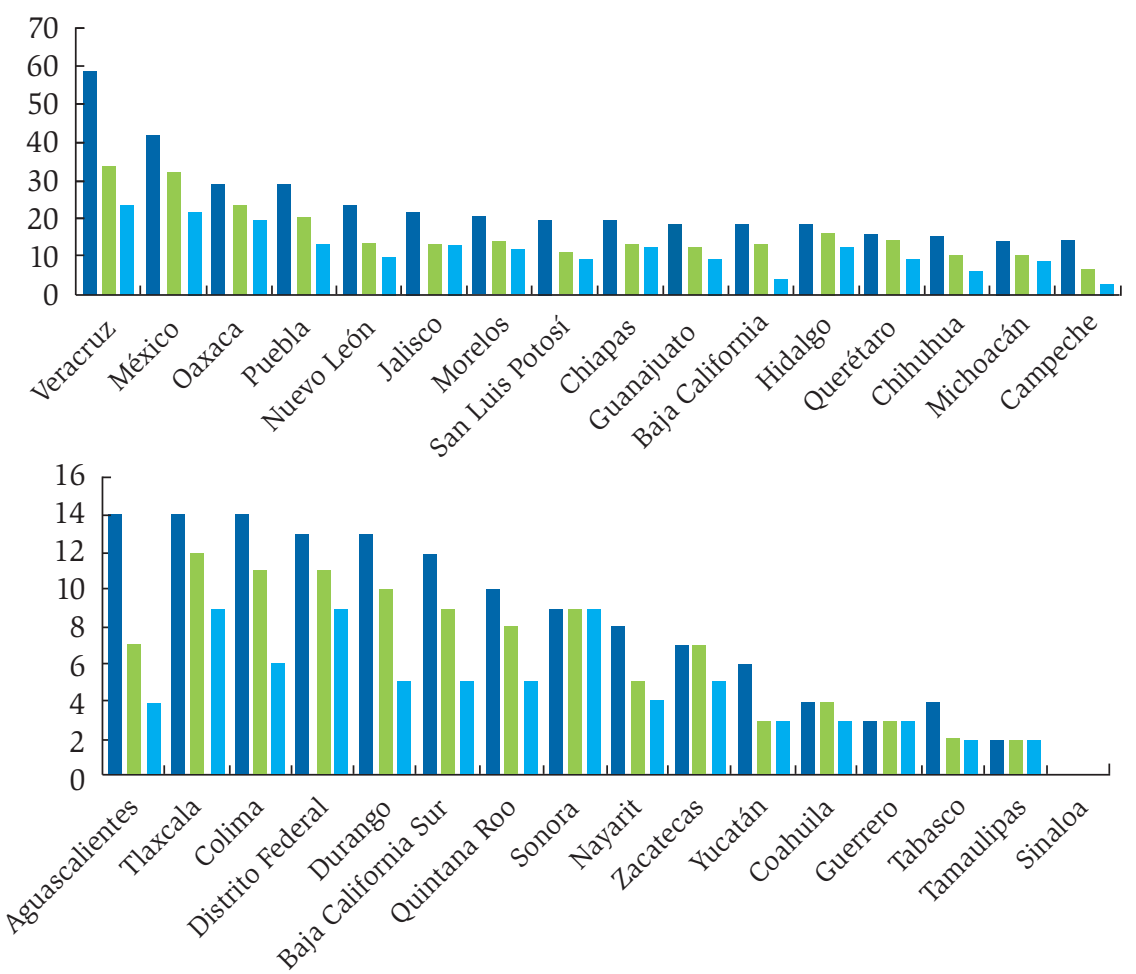

- Campamentos totales $\square$ Localidades con campamentos $\square$ Municipios con campamentos

Fuente: Elaboración propia con base en Frausto Martínez y Nava Jiménez (s.f.).

Gráfica 1. Concentración de los campamentos. 


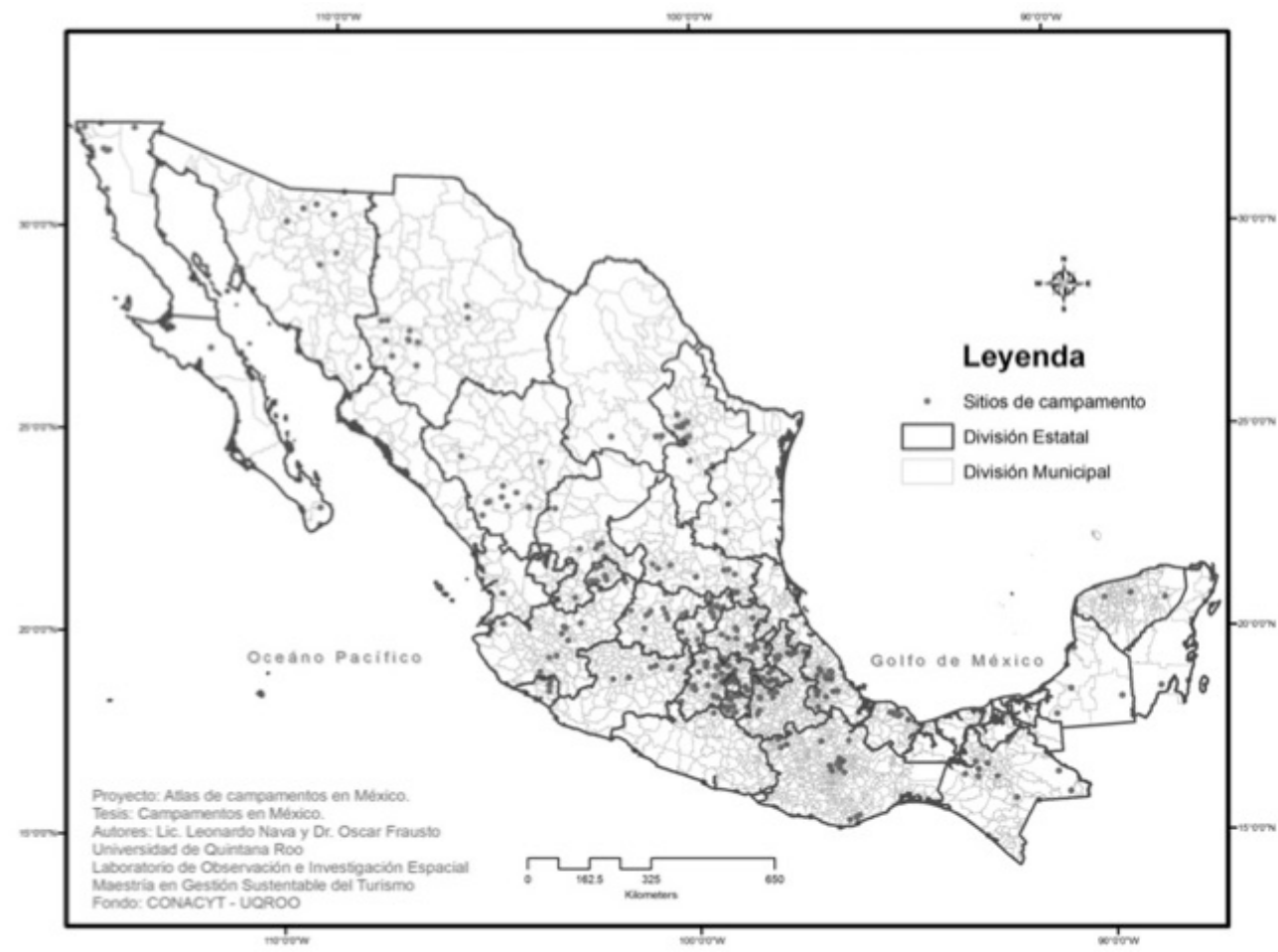

Figura 1

\section{Caracterización}

\section{Altitud}

La mayor concentración de campamentos está en la zona centro de México, dentro del eje volcánico central, en un sistema montañoso entre los 2000 y los 2500 metros sobre el nivel del mar (msnm) (figura 2). En los relieves inferiores a 200 msnm, se observa una distribución de campamentos más espaciada, aunque pueden advertirse nodos específicos sobre líneas costeras, como la Riviera Maya (Quintana Roo), Campeche (Campeche) y el corredor Rosarito-EnsenadaSan Lucas (Baja California-Baja California Sur). En áreas con altitudes mayores a los $3500 \mathrm{msnm}$, la presencia de campamentos es a manera de nodos, como los de Santiago (Monterrey), la reserva natural El Ocote (Chiapas) y la Sierra Juárez (Oaxaca). 


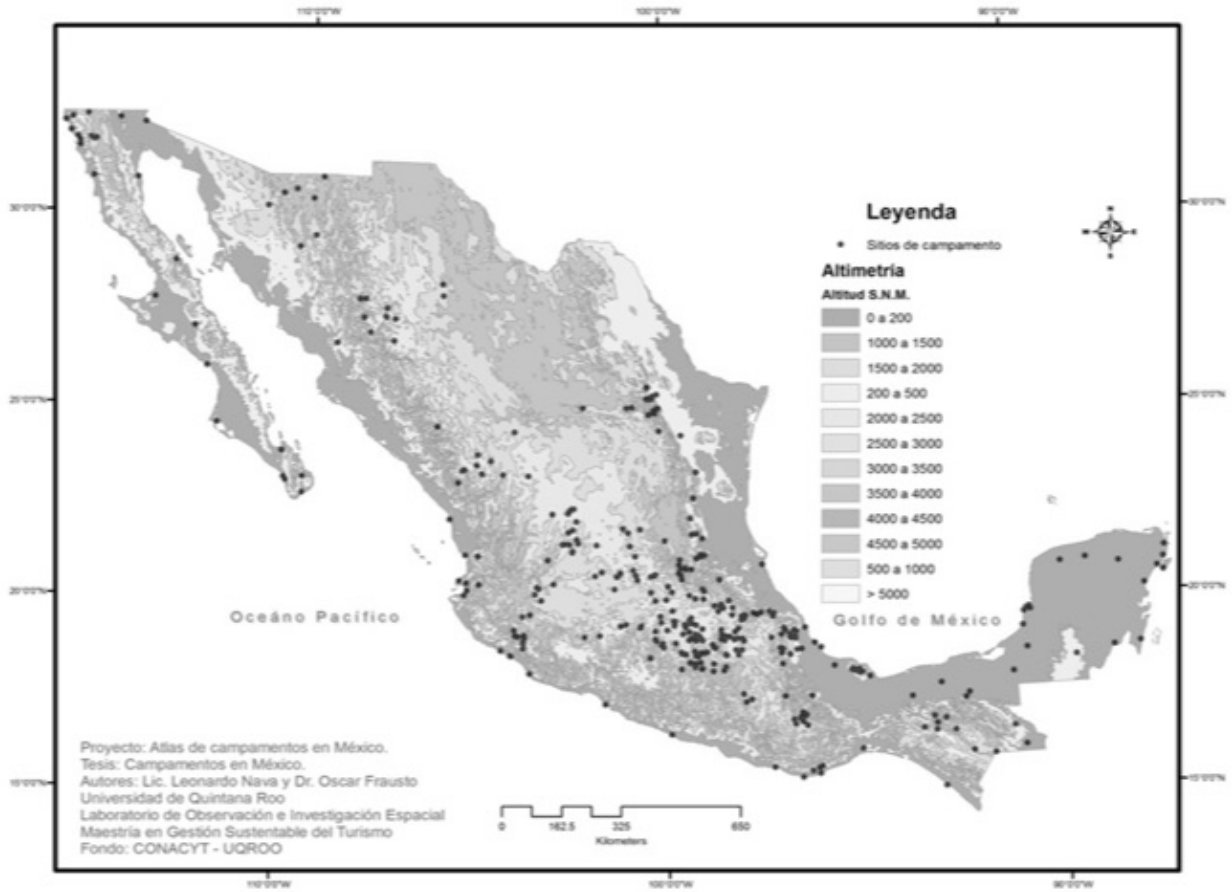

Fuente: Atlas de campamentos en México 2014.

Figura 2. Ubicación de los campamentos en relación con las zonas altimétricas

\section{Áreas naturales protegidas}

Los sitios de campamentos dentro de las AnP son una de las ofertas principales de este sector por el atractivo natural que proporcionan. En el centro del país, en las inmediaciones del Distrito Federal, en el Estado de México y en Morelos, se congregan una serie de pequeñas ANp. Esta concentración de espacios protegidos, en la zona con mayor densidad poblacional en México, también es el área con mayor cantidad de campamentos.

Otra aglomeración similar de pequeñas ANP se encuentra entre los estados de Nayarit, Jalisco, Zacatecas y Durango; sin embargo, en este conglomerado no existe presencia considerable de actores: al contrario que en la zona centro, estos se ubican en su periferia. 
Por su parte, en el estado de Nuevo León, en el noreste del país, se presenta un escenario distinto: existe una zona uniforme, protegida por una ANp de mayor tamaño con una concentración de campamentos en su interior.

En los extremos sureste y noroeste de México se localizan las ANP más extensas, pero los campamentos ahí ubicados son escasos. Se observa un patrón general de los actores establecidos en los límites de las mismas (figura 3).

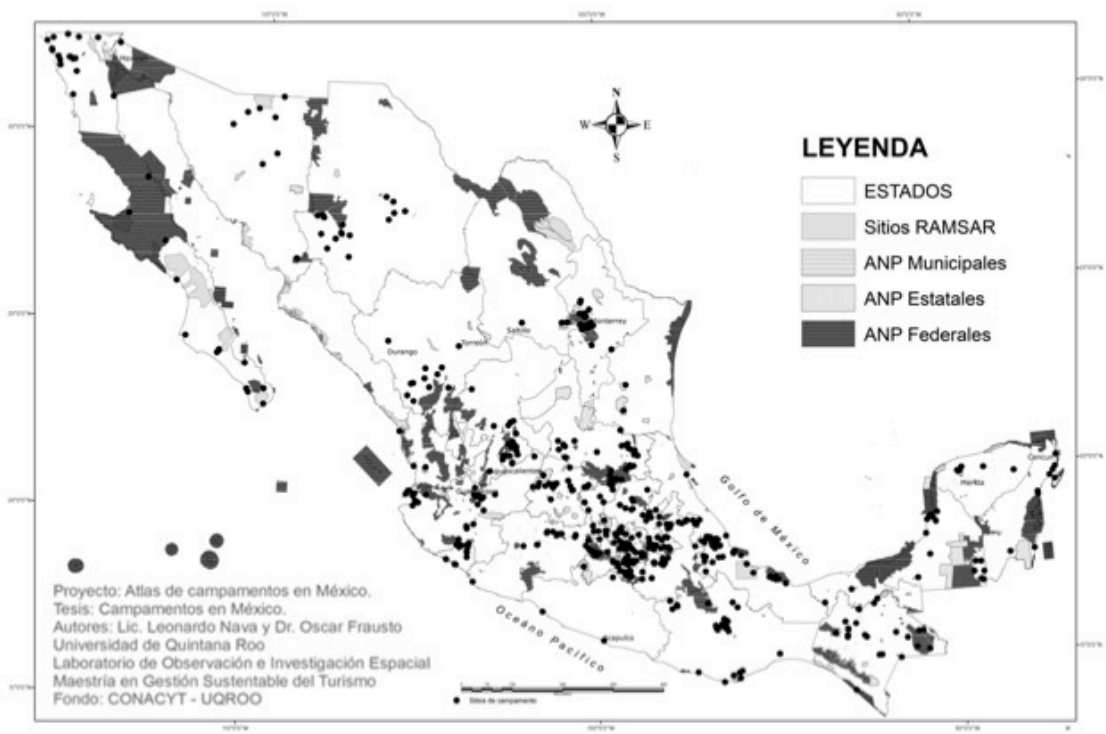

Fuente: Atlas de campamentos en México 2014.

Figura 3

\section{Atractivo natural}

Conjugando elementos de su hipsometría, clima y asociación vegetal, se resume un sistema de paisajes característico que puede ser considerado determinante para la oferta. La montaña (de húmeda a muy seca, de bosque de coníferas y vegetación xerófita) es uno de los paisajes donde se encuentra el mayor número de sitios de campamento. En proporción, son mínimos y dispersos los establecimientos que se localizan fuera del relieve de montaña. Casi todos los tipos de relieves han sido utilizados para la instalación de campamentos, excepto los de las llanuras de bosque de coníferas y espinoso (figura 4). 


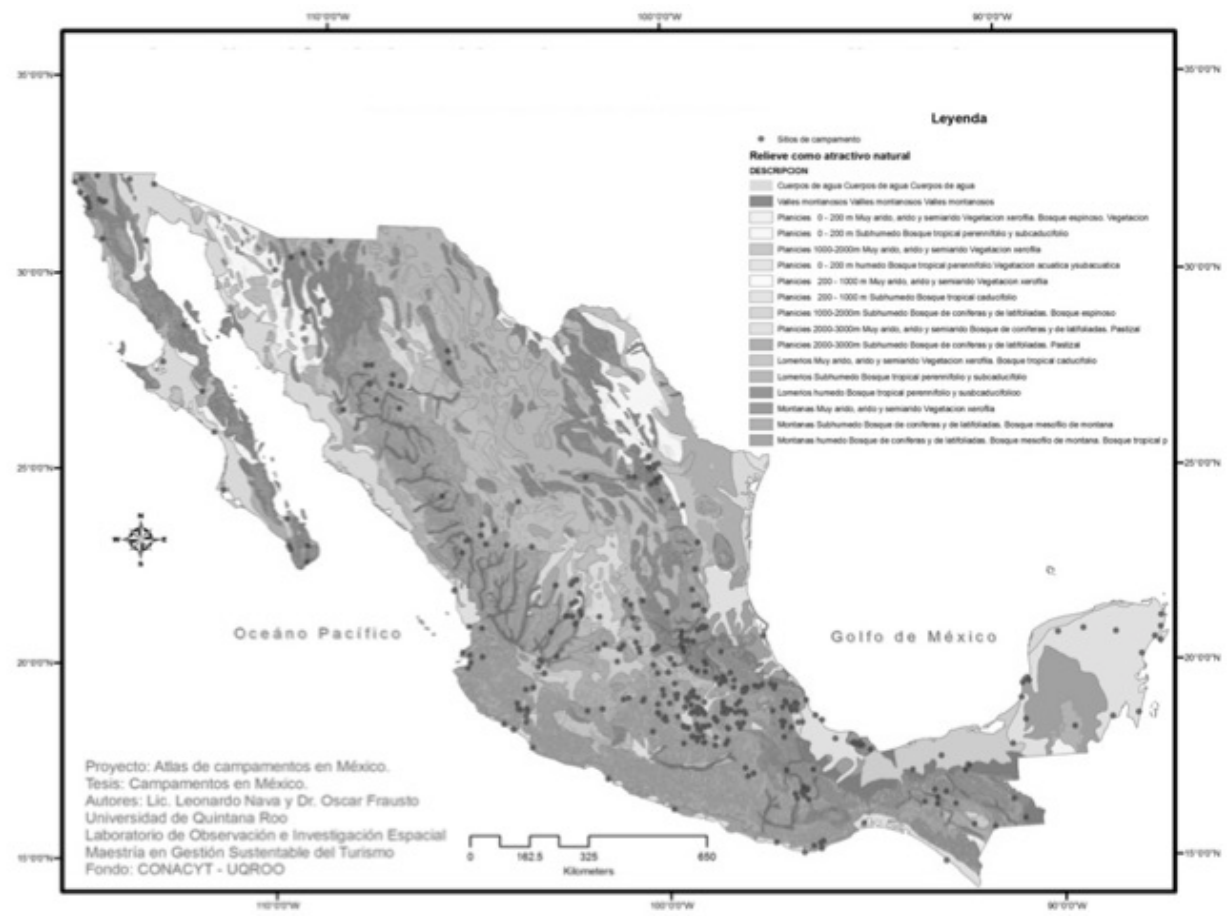

Figura 4

Distribución en la red de carreteras y sistema de localidades

Mediante la herramienta de análisis de proximidad del software ArcGIS 9.3, se relacionaron las bases de datos de los campamentos en México y la del sistema carretero nacional (Digital Chart of the World, 1985). Se establecieron cuatro niveles de cercanía, en cientos de kilómetros. El resultado es el modelo de la figura 5, que permite determinar la red de conexión entre los núcleos de actores.

Las vías interestatales que conectan la zona centro con los estados de Guanajuato, Querétaro, Michoacán, San Luis Potosí, Hidalgo y Veracruz presentan claramente corredores sobre los que se han instalado campamentos, desahogando parte del flujo de la zona centro. Otro corredor de campamentos es el generado a lo largo de las vías que vinculan dos núcleos urbanos principales: de Zacatecas a Manzanillo; se trata de caminos que enlazan el sector centro occidente con la costa. 
La conectividad internacional es clave para los corredores de campamentos Rosarito-Ensenada y Ciudad Juárez-Chihuahua, ya que permite un flujo de visitantes proveniente de Estados Unidos; efecto que no se repite en el resto de las fronteras norte y sur (figura 5).

Finalmente, cabe destacar el sistema de red del valle central de Chiapas, que bordea el macizo de la selva Lacandona.

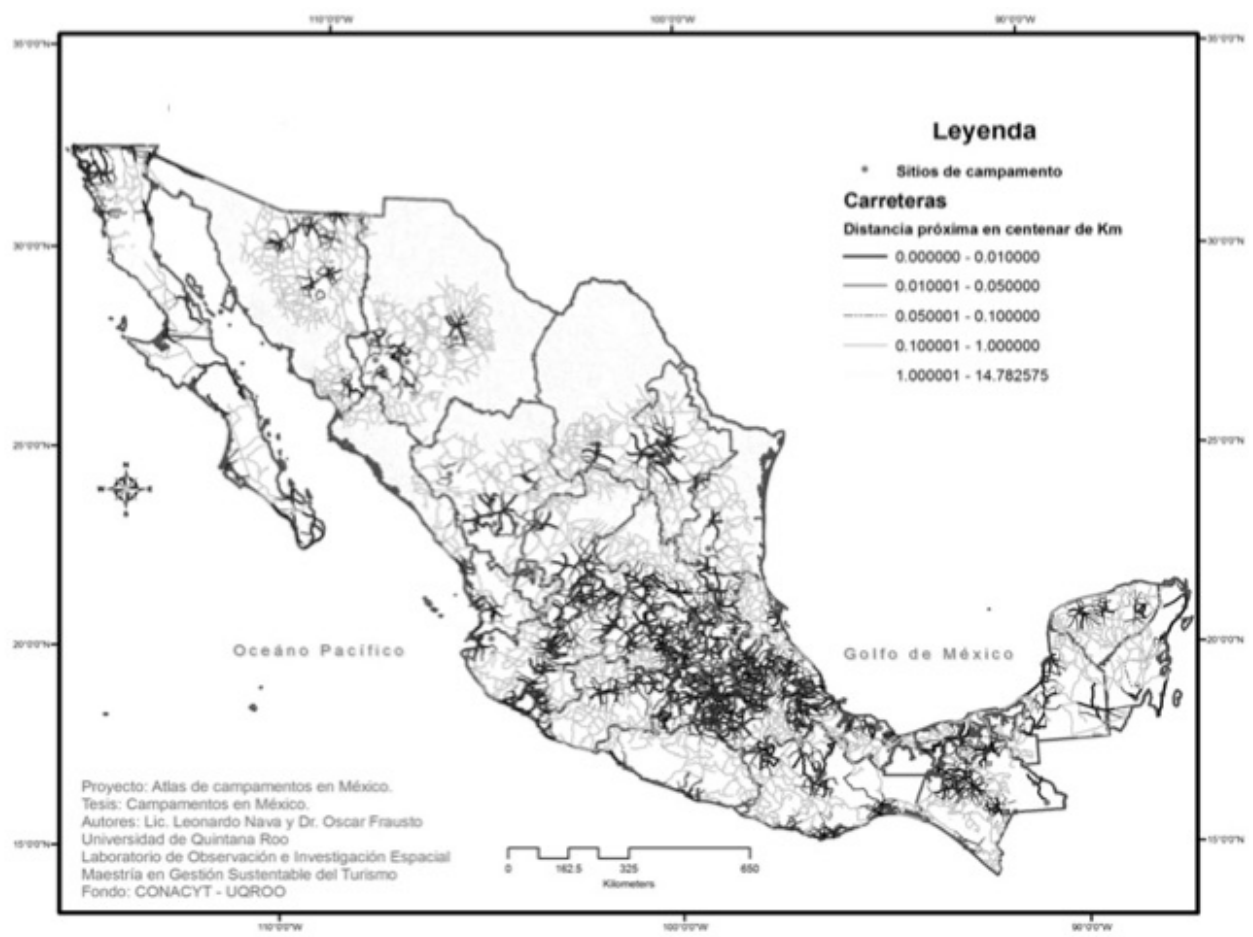

Figura 5 


\section{Discusión}

Los resultados de las entrevistas evidenciaron una falta de consenso y reflexión para el uso del término campamento. El concepto varía según el discurso de cada actor. Aquellos denominados campamentos organizados atienden a un público infantil y argumentan su operación con una finalidad formativa; ${ }^{1}$ muy distinto de los que se reconocen como turísticos, cuyo objetivo es el desarrollo económico. ${ }^{2}$ Cada tipo de campamento muestra particularidades en su infraestructura; por ejemplo, los organizados presentan mayor cantidad de comodidades para la estadía de los visitantes, mientras que los turísticos tienen instalaciones sencillas y facilidades limitadas.

En cuanto a la metodología utilizada, la encuesta en línea permitió recolectar datos de campamentos distribuidos en todo el territorio nacional; 81 por ciento reafirmó su localización, se depuró la base de datos al determinar aquellos que nunca han ofrecido o ya no ofrecían su espacio como campamento (12 por ciento de los encuestados); 7 por ciento no respondió sobre la localización. Uno de los elementos significativos fue el señalamiento de las limitantes para su establecimiento: violencia, delincuencia organizada, mala imagen del destino y falta de apoyos financieros, lo cual se refleja en los escasos actores en los estados de Sinaloa, Tamaulipas y Coahuila.

La necesidad de esparcimiento de las personas que habitan los grandes núcleos urbanos, alejados del entorno natural, ha generado una demanda de actividades de recreación cercanas a su residencia. La proliferación de campamentos en las zonas colindantes al Distrito Federal y a las grandes manchas urbanas del Estado de México puede ser una respuesta para satisfacer esta necesidad en un ambiente natural. Por lo general, los pobladores del área metropolitana tienen que salir de su entidad para encontrar los espacios naturales con los que ya no cuentan en su localidad, y la distancia que deben recorrer es cada vez superior, por lo que este viaje requiere una mayor inversión de tiempo y recursos, lo que reduce el flujo de visitantes, pero, al mismo tiempo, aumenta su demanda de servicios como hospedaje y alimentación. Esto se confirma al analizar la estructura de la red carretera y su relación con los sitios de campamento.

${ }^{1}$ Entrevista grabada a V. L. Vergara, 8 de julio de 2013.

${ }^{2}$ Entrevista grabada a R. Chambor, 24 de octubre de 2013. 
Se reconocen diferentes formas de campamentos de acuerdo con la demanda, que en un primer plano atiende la exigencia de hospedaje de una manera alternativa al servicio de hospitalidad hotelera. Además, los campamentos han ido incorporando una amplia gama de servicios (cabañas, habitaciones, albercas, internet inalámbrico, entre otros) y actividades (senderismo, tirolesa, ciclismo de montaña, etc.), lo cual ha motivado que se ponga en duda que los campamentos sean considerados como pertenecientes exclusivamente al ecoturismo (Carballo Sandoval, 2001; Semarnat, 2006) o al turismo de naturaleza (Sectur, 2013). Dada la evolución de los actores, puede decirse que en la actualidad algunos son más parecidos a establecimientos hoteleros que a los campamentos en su concepción tradicional (Brooker y Joppe, 2013).

En cuanto a las Anp, la presencia de campamentos puede actuar como indicador de su vocación. Por ejemplo el conglomerado de ANP del Estado de México y Morelos, y el de anp de Nuevo León, donde los campamentos se concentran dentro de áreas pequeñas y medianas. Por otro lado, la escasa presencia de campamentos dentro de las ANP más grandes del territorio nacional, y a la vez más alejadas del centro de México, podría sugerir una vocación mayormente dirigida hacia la conservación o a otros fines no recreativos.

\section{Conclusiones}

En México se reconocen 519 campamentos oficiales ubicados en 363 localidades, concentrados cerca de los centros urbanos, en núcleos con alta densidad de actores. Cada nodo agrupa campamentos caracterizados de forma específica, tanto por el área urbana que atiende como por la finalidad con la que opera.

El estudio de los campamentos a través de su estructura (actores, flujos, estructura y jerarquía) ha sido poco tratado en México, pero permite un debate actual en torno a los límites de la actividad por medio de diversas aproximaciones. En este trabajo se revisa el carácter territorial respecto a los sistemas naturales para evidenciar la existencia de un sistema de campamentos, lo cual resulta relevante si se busca un desarrollo sustentable del mismo.

Se destaca la presencia de la actividad con patrones territoriales y regionales que no han sido reportados. Con todo, su institucionalidad es evidente al ser parte de asociaciones y grupos que incentivan este sector, en especial la Secretaría 
de Turismo, la Asociación Mexicana de Campamentos y la International Camping Fellowship.

\section{Agradecimiento}

El presente trabajo se desprende de la tesis de maestría en Gestión Sustentable del Turismo, en el Laboratorio de Observación e Investigación Espacial de la Universidad de Quintana Roo. Se agradece el apoyo financiero del Consejo Nacional de Ciencia y Tecnología de México.

\section{Fuentes consultadas}

Amc (2013). Directorio de los miembros de la Asociación Mexicana de Campamentos. México: Asociación Mexicana de Campamentos.

Aparicio, M. (1999). Aire libre: un medio educativo. 2a ed. Madrid: Editorial ccs. Asouti, E. (2003). "Woodland Vegetation and Fuel Exploitation at the Prehistoric Campsite of Pinarbası, South-Central Anatolia, Turkey: The Evidence from the Wood Charcoal Macro-Remains". Journal of Archaeological Science, 30 (9), 1185-1201. http://doi.org/10.1016/S03054403(03)00015-3

Brooker, E. y M. Joppe (2013). “Trends in Camping and Outdoor Hospitality. An International Review”. Journal of Outdoor Recreation and Tourism, 3-4, 1-6. http://doi.org/10.1016/j.jort.2013.04.005

Carballo Sandoval, A. (2001). "Concepción y perspectiva del ecoturismo en México”. Doctrina, 52, noviembre-diciembre, 83-86.

Conabio (2012). Portal de Geoinformación, Sistema Nacional de Información Sobre Biodiversidad. Disponible en: http://www.conabio.gob.mx/informacion/gis/ [2015, 15 de febrero].

Digital Chart of the World (1985). "Red de carreteras" [en línea]. Catálogo de metadatos geográficos. México: Comisión Nacional para el Conocimiento y Uso de la Biodiversidad. Disponible en: file://C:/Users/ Leo \%20Polo/Desktop/Maestria/Tesis \% 20CAMPISMO/SIG/Conabio/ Carreteras/carre1mgw.html 
Feo Parrondo, F. (2003). “Los campings en España”. Cuadernos de Turismo, 11, enero-junio, 83-96.

Frausto Martínez, O. y L. Nava Jiménez (s.f.). Atlas de campamentos en México. Cozumel: inédito.

Grupo Intercom (2013). yumping.com.mx. Campamentos. Disponible en http:// www.yumping.com.mx/campamentos [2015, 15 de febrero].

Heritage Appreciation Unit (2006). Review of Camping Opportunities in New Zealand. Report to the Minister of Conservation. Wellington: Department of Conservation Te Papa Atawhai.

Hiernaux, D. (1989). "El espacio reticular del turismo en México”. Geografía y Desarrollo, 2 (3), 31-39.

ICF (2013). "Links to Organizations and Camps-Current ICF Members” [en línea]. International Camping Fellowship. Disponible en: http://www.campingfellowship.org/ICF-Web/DesktopDefault.aspx?tabid = 51

(2014). ICF Members as of March 1st 2014 (p.15). Disponible en: http:// www.campingfellowship.org/upload/file/icf-members-as-of-march1st-2014.pdf [2015, 15 de febrero].

Janiskee, R.L. (1998). "Resort Camping in America”. Annals of Tourism Research, 17 (3), 385-407.

Lee Nolan, M. y T. Broxon (2000). “Camping”, en Jafar Jafari (ed.). Encyclopedia of Tourism. Londres/Nueva York: Routledge, 65-66.

Leefers, L.A. y J.M. Vasievich (1999). "An Analysis of Campground Resources in the Lake States” [en línea]. Reporte. Great Lakes Ecological Assessment-North Central Research Station. Disponible en: http://www. ncrs.fs.fed.us/gla/reports/LSCamps.PDF

López Palomeque, F. (1988). “Geografía del turismo en España: una aproximación a la distribución espacial de la demanda turística y de la oferta de alojamiento”. Documents D’annàlisi Geogràfica, 13, 35-64.

Luján Ferrer, E.M. y K. Rodríguez Sánchez (2011). "El campamento como programa didáctico: hacia una propuesta teórico-metodológica para su implementación en los museos”. Revista Actualidades Investigativas en Educación, 11 (1), 1-33.

McEwen, D.N. (1986). "Recreation Quality and the Market for Tent Camping”. Journal of Park and Recreation Administration, 4 (2), 83-95. 
México Desconocido (2005). "Campamentos en México”. Guía México Desconocido, 96 (ed. especial), agosto.

Mexplora (s.f.). Campamentos recomendados en México [en línea]. Disponible en: http://www.campamentosmexico.com/ [2015, 15 de febrero].

Miranda Montero, M.J. (1985). "El camping. La forma más reciente de turismo". Cuadernos de Geografía, 37, 157-174.

Ortiz, E. A. (1994). "El campismo mexicano actual", en V.L. Vergara (ed.). Memorias del $1^{\circ}$ Congreso Mundial de Recreación.(pp. 18-26). México, D. F.: Asociación Mexicana de Recreación.

Presidencia de la República (1984). "Reglamento de establecimientos de hospedaje, campamentos y paraderos de casas rodantes”. Diario Oficial de la Federación, 20 de agosto, 110-115.

Ramsing, R. (2007). “Organized Camping: A Historical Perspective”. Child and Adolescents Psychiatric Clinics of North America, 16 (4), 751-754. http://doi.org/10.1016/j.chc.2007.05.009

Sectur (2010). "Norma Oficial Mexicana Nom-06-TuR-2009, Requisitos mínimos de información, higiene y seguridad que deben cumplir los prestadores de servicios turísticos de campamentos". Diario Oficial de la Federación, 6 de septiembre, 104-115.

(2013). "Directorio de Prestadores de Servicios Turísticos de Naturaleza" [en línea]. México: Secretaría de Turismo. Disponible en: http://www. sectur.gob.mx/es/sectur/sect_Directorio_de_Prestadores_de_Servicios_de_Tur [2012, 26 de octubre].

Semarnat (2006). Introducción al ecoturismo comunitario. 2a ed. México: Secretaría de Medio Ambiente y Recursos Naturales.

Varela Merino, E. (2009). Los galicismos en el español de los siglos XVI y XVII. Vol. II. Madrid: Consejo Superior de Investigaciones Científicas (Anejos de la Revista de Filología Española, 100).

Vergara, V. (1994). "La recreación, un camino de esperanza en el futuro de México”, en V.L. Vergara (ed.). Memorias del $1^{\circ}$ Congreso Mundial de Recreación. (pp. 2-10). México, D.F.: Asociación Mexicana de Recreación. yumping.com.mx. Turismo de aventura y deportes extremos (s.f.). Campamentos [en línea]. Disponible en: http://www.yumping.com.mx/campamentos 